\title{
NY-ESO-1 Protein/Microparticle MDP/Bacterial DNA-containing MIS416 Vaccine
}

National Cancer Institute

\section{Source}

National Cancer Institute. NY-ESO-1 Protein/Microparticle MDP/Bacterial DNAcontaining MIS416 Vaccine. NCI Thesaurus. Code C128887.

A combination preparation composed of a protein derived from the human tumorassociated antigen (TAA) cancer-testis antigen 1 (NY-ESO-1) and a microparticle combining two immune-modifying components derived from the bacterium Propionibacterium acnes, a bacterial cell wall component that is rich in muramyl dipeptide (MDP) and bacteria-derived single-stranded DNA fragments, with potential immunomodulating, immunoadjuvant and antineoplastic activities. Upon administration of NY-ESO-1 protein/microparticle MDP/bacterial DNA-containing MIS416 vaccine, MIS416 localizes in and is taken up mainly by the liver, thereby forming a liver depot. MIS416 is then taken up by immune cells, such as monocytes and dendritic cells (DCs), where MDP and the bacterial DNA target and bind to the cytosolic innate pattern recognition receptors (PRRs) nucleotide-binding oligomerization domain-containing protein 2 (NOD2), and toll-like receptor 9 (TLR9), respectively. The simultaneous binding and activation of both NOD2 and TLR9, leads to activation of both NOD2 and TLR9 signaling pathways. This stimulates the innate immune system, induces secretion of cytokines, particularly interferon (IFN), and modulates the activation of various immune cells. In the presence of the NY-ESO-1 peptide, MIS416 enhances the cytotoxic Tlymphocyte (CTL)-mediated immune response against NY-ESO-1, resulting in an increased anti-tumor immune response. NY-ESO-1 is expressed in normal testes and on the surfaces of various tumor cells, and plays a key role in tumor cell proliferation and survival. 